\title{
lodine I 131 Anti-Fibronectin Antibody Fragment L19-SIP
}

National Cancer Institute

\section{Source}

National Cancer Institute. Iodine I 131 Anti-Fibronectin Antibody Fragment L19-SIP. NCI

Thesaurus. Code C91389.

An iodine 131 radioimmunoconjug ate of a small immunoprotein (SIP), derived from the variable region fragment of human monoclonal antibody L19, that is directed against the extra-domain B (ED-B) of fibronectin, with potential radioimmunotherapeutic activity. The SIP moiety of iodine I 131 anti-fibronectin antibody fragment L19-SIP binds to the ED-B domain of fibronectin on tumor cells in the tumor neovasculature. Upon internalization, the I 131 radionuclide may selectively detect or deliver cytotoxic radiation to fibronectin-expressing tumor cells. ED-B of fibronectin, a naturally occurring marker of tissue remodeling and angiogenesis, is expressed in the majority of aggressive solid human tumors, whereas it is not detectable in normal vessels and tissues. 